\title{
A THEOREM ON DISKS
}

O. G. HARROLD, JR.

This paper is concerned with the question of whether or not, if an arbitrary simple closed curve $J$ in three space is given, there exists a disk (closed, topological 2-cell) $D$ such that (i) $D$ and $J$ meet in a single point interior to $D$, and (ii) the boundary of $D$ links $J$. Our interest derives from the fact that in characterizing tame simple closed curves [3] such disks exist in abundance. In fact it may be required that the disk in question is locally polyhedral save where it meets $J$. Since the property of being locally polyhedral is not an invariant under homeomorphisms of three space on itself, it is not clear that if this extra condition were imposed that an invariant property would be obtained. It turns out that with this condition added one does obtain an invariant property as may be shown by an appeal to Moise's general interpolation theorem as indicated in [2]. However, it seems not to be known whether the class of curves admitting such disks is the class of all curves in three space or not. Thus we are led to consider the more general problem above.

The homologies implied below in the discussions of linking may be taken modulo 2. An arc is called semi-rectifiable if in some topological representation of the unit interval on it one of the co-ordinate functions is of bounded variation. ${ }^{1}$

THEOREM. If there is a homeomorphism of space on itself carrying $J$ on $J^{1}$ such that for some pair of points $a$ and $b$ of $J^{1}$, one of the arcs of $J^{1}$ determined by $a$ and $b$ is semi-rectifiable, then there exists a tame disk $D$ such that (i) and (ii) hold.

Proof. Let $A$ be the arc of $J^{1}$ that is semi-rectifiable. If any plane contains a nondegenerate connected subset of $A$ (hence of $J^{1}$ ), the given curve contains a tame sub-arc and the solution, as mentioned above, is immediate. Hence we take the co-ordinate function along $A$ which is of bounded variation to be nonconstant. If $x_{1}$ is this coordinate, let $\pi$ be a plane $x_{1}=$ constant that separates $a$ and $b$,

Received by the editors January 4, 1955.

1 The author is indebted to W. S. Snyder for pointing out that the original assumption of rectifiability may be replaced by semi-rectifiability.

R. H. Bing has discovered an example of a simple closed curve that fails to have properties (i) and (ii) for any disk. This example will appear in Journal de Mathematiques Pures et Appliquees. 
where $a$ and $b$ lie on $A$. Evidently $\pi \cap A$ is not vacuous and there is a point $p$ of $\pi \cap A$ that separates $a$ and $b$ on $A$. By the bounded variation of the $x_{1}$ co-ordinate along $A$, there are uncountably many planes $\pi^{1}$, parallel to $\pi$, as near $\pi$ as we please, and so that $A \cap \pi^{1}$ is a finite set [1]. It is no loss to take $\pi^{1}$ so that no maximum nor minimum of $x^{1}$ lies in $\pi^{1}$. By the finiteness of $A \cap \pi^{1}$, there is a disk $D^{*}$ in $\pi_{1}$ with center at $q \in A \cap \pi^{1}$ so that $D^{*} \cap A=D^{*} \cap J^{1}=(q)$. The boundary of $D^{*}$ evidently links $J^{1}$, since the plane $\pi^{1}$ contains no maximum nor minimum of $x_{1}$. If $h$ is the given homeomorphism, the sought for disk $D$ is given by $D=h^{-1}\left(D^{*}\right)$.

\section{REFERENCES}

1. S. Banach, Sur les lignes rectifiables, Fund. Math. vol. 7 (1925) p. 228.

2. O. G. Harrold, Jr., The enclosing of simple arcs and curves by polyhedra, Duke Math. J. vol. 21 (1954) pp. 615-622.

3. O. G. Harrold, Jr., H. C. Griffith, and E. E. Posey, A characterization of tame curves in three-space, Proc. Nat. Acad. Sci. U.S.A. vol. 40 (1954) pp. 235-237.

The UNIVERSITY OF TENNESSEE 